\title{
A symmetry breaking mechanism for epithelial cell polarization
}

\author{
A. Veglio, ${ }^{1,2}$ A. Gamba, ${ }^{3,2}$ M. Nicodemi ${ }^{4}$ F. Bussolino, ${ }^{1}$ and G. Serini ${ }^{1}$ \\ ${ }^{1}$ Department of Oncological Sciences and Division of Molecular Angiogenesis, \\ Institute for Cancer Research and Treatment, University of Torino School of Medicine, Candiolo (TO), Italy. \\ ${ }^{2}$ CNISM, Corso Duca degli Abruzzi 24, 10125 Torino, Italy. \\ ${ }^{3}$ Politecnico di Torino, Corso Duca degli Abruzzi 24, 10125 Torino, Italy; and INFN Torino, Italy. \\ ${ }^{4}$ Dept. of Physics \& Complexity Sciences, University of Warwick, UK; and INFN Napoli, Italy.
}

\begin{abstract}
In multicellular organisms, epithelial cells form layers separating compartments responsible for different physiological functions. At the early stage of epithelial layer formation, each cell of an aggregate defines an inner and an outer side by breaking the symmetry of its initial state, in a process known as epithelial polarization. By integrating recent biochemical and biophysical data with stochastic simulations of the relevant reaction-diffusion system we provide evidence that epithelial cell polarization is a chemical phase separation process induced by a local bistability in the signaling network at the level of the cell membrane. The early symmetry breaking event triggering phase separation is induced by adhesion-dependent mechanical forces localized in the point of convergence of cell surfaces when a threshold number of confluent cells is reached. The generality of the emerging phase separation scenario is likely common to many processes of cell polarity formation.
\end{abstract}

\section{INTRODUCTION}

The development of epithelial tissues (e.g., kidney tubules, respiratory and gastrointestinal tracts, etc.) results from complex morphogenetic processes implying the arrangement of cells in layers organized along specific directional axes 1, 2]. Epithelial cells are endowed with a self-polarization mechanism defining an 'inner' and 'outer' side, which is mandatory to allow organs to exert their vital functions. In a well established in vitro cell system, which recapitulates the in vivo morphogenesis, after a single epithelial cell is seeded in a threedimensional gel (Fig. 17), cell division begins, and a multicellular aggregate arises 1. The cells in the aggregate are bound each other (through cadherin molecules, Fig. 1b) and to an extracellular matrix (through integrin molecules, Fig. 17). When the cell number reaches 5-6 units, an inner cavity, named lumen, is spontaneously opened [3] (Fig. 17,b). Afterwards, cells develop a top (called apical) and a bottom (basolateral) side (Fig. 1 d) having different chemical features, while cell-cell and cellmatrix contacts only persist in the basolateral region. Finally, the border between apical and basolateral sides is sealed by ring-shaped tight junction proteins, which spontaneously find their functional position (Fig. 1 $\mathrm{d}$ ) and prevent intermixing of chemical components between the apical and the basolateral membranes, as well as the outpouring of liquids from the lumen. The full polarization process has a complex nature and involves different factors and stages. However, recent experiments have determined its master regulator (see [4, 5] and ref.s therein): intracellular asymmetry and lumen opening are controlled by PIP2/3 phospholipids (see Fig 2 ) and their interaction with PTEN/PI3K enzymes which induce PIP2/3 segregation to opposite poles, while the PAR complex further stabilizes axial polarity (see the review [1] and ref.s below). Even in such an in vitro system, however, the mechanism whereby the cell original spatial symmetry is spontaneously broken and polariza- tion develops remains mysterious [1.

To describe cell differentiation, polarization and signal localization mechanisms, stochastic reaction-diffusion [ 6 and coupled kinetic rate equations have been widely used for intracellular signaling, gene regulation and autocatalytic reaction systems (see e.g. [7] 9 and ref.s therein). In this context, here we use reaction-diffusion equations to model the PIP2/3 master regulator in order to address some open questions [1], namely the mechanisms for: i) the lumen site choice; ii) its opening; iii) the control of its final size; $i v$ ) the localization of tight junctions. After delineating the chemical reactions involved in the process, by simulation of its master equation we show that self-polarization can be understood in terms of statistical physics concepts as a symmetry breaking mechanism driven by the chemical regulatory network. We finally interpret the simulation results in terms of a simple mean-field model.

\section{MODEL}

Recent experiments have shown that PIP2 and PIP3 for a module that acts as a master regulator controlling all signaling pathways and cytoskeletal dynamics required for epithelial cell polarization 4, 5. PIP2/PIP3 levels are regulated by the counteracting enzymes PI3K and PTEN, which respectively catalyze the switch of PIP2 to PIP3 and vice versa 1, 10 (Fig. 2). The phospholipids (PIP2/3) are stably localized in the inner face of the cell membrane where they diffuse. The enzymes (PI3K/PTEN) diffuse instead in the cell volume, where they are present in limiting amounts, and become active upon association with membrane spanning proteins or lipids. PTEN levels in the membrane are controlled by its binding to PIP2, thus realizing a positive feedback loop (see Fig. 2). PI3K levels in the membrane are controlled by its binding to cell-cell adhesive receptors (cadherins) and cell matrix adhesive receptors (inte- 


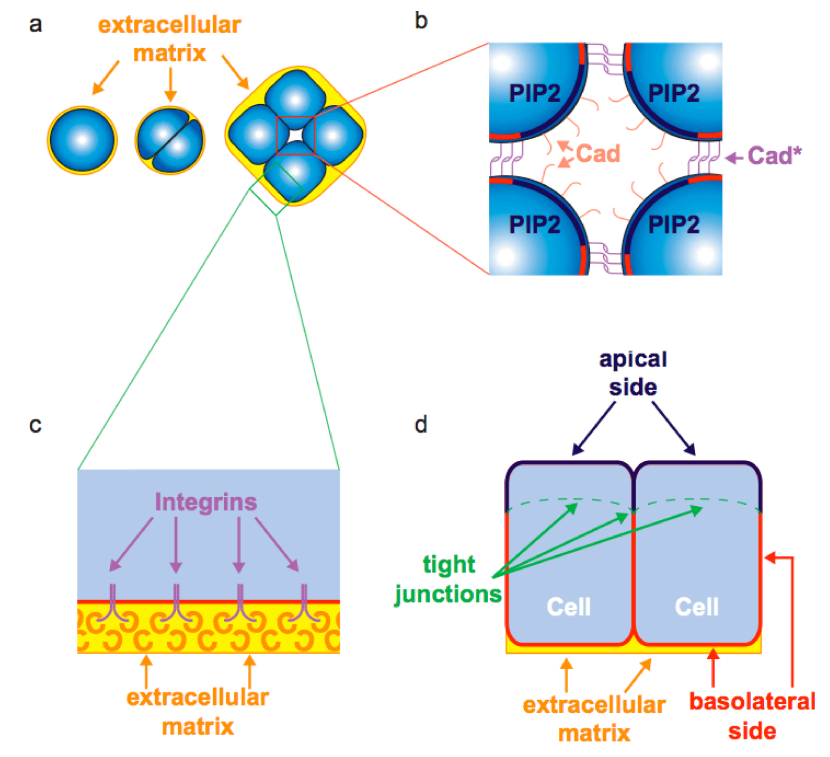

FIG. 1: (Color online) a,b) After a few cell divisions, at the common point of contact among the cells of an aggregate the intrinsic high membrane curvature locally induces formation of a microlumen (inner circle) and the breaking of cell-cell adhesive receptor links (pink and purple bars). This breaking favors the appearance of a small region rich in PIP2 lipids (blue) which provides a nucleation center for the ensuing symmetry breaking process. b,c) Everywhere but in correspondence of the PIP2-rich region, the cell is surrounded by adhesive contacts, either cell-cell (cadherins) or cell-matrix contacts (integrins). Cad (pink bars) and $\mathrm{Cad}^{*}$ (purple bars) respectively indicate inactive and active cadherins. d) Scheme of the apical (blue) and basolateral (red) regions and the ring of tight junctions (green) spontaneously assembling at their interface. The extracellular matrix (yellow) is indicated as well.

grins, schematically indicated by $\mathrm{C} / \mathrm{M}$ in Fig. 2] [11. To bind PI3K, cadherins must be activated $\left(\mathrm{Cad}^{*}\right.$ in Fig. 1p and 2p by engagement with cadherins of a neighboring cell (named $\mathrm{C} / \mathrm{C}$ in the diagram of Fig. 2) PI3K is active only when associated to either activated cadherins or integrins. Since PIP3 stabilizes the activated form $\mathrm{Cad}^{*}$ [12, these interactions create a positive PIP3PI3K feedback loop, mediated by the existence of cellcell contacts (Fig. 22). Before polarization, cadherins and integrins are activated along the whole membrane and PIP3 uniformly prevails on PIP2 determining a stable PIP3-rich phase over the whole membrane. A local depletion of PIP3-PI3K can be created if a large enough membrane area with disrupted cell-cell links is formed, thereby breaking the PIP3-PI3K feedback loop (Fig. 2) and originating a germ of a PIP2-rich phase (Fig. $1 \mathrm{~b}$ and 2). Then, the PIP2-PTEN feedback loop may locally prevail, inducing a PIP2 and PIP3 surface compartmentalization that splits the cell membrane in two regions, or phases [13], characterized by different chemical concentrations of the signaling molecules.

Several mechanisms have been proposed 4 to explain the initiation of the polarization process, which is typ-

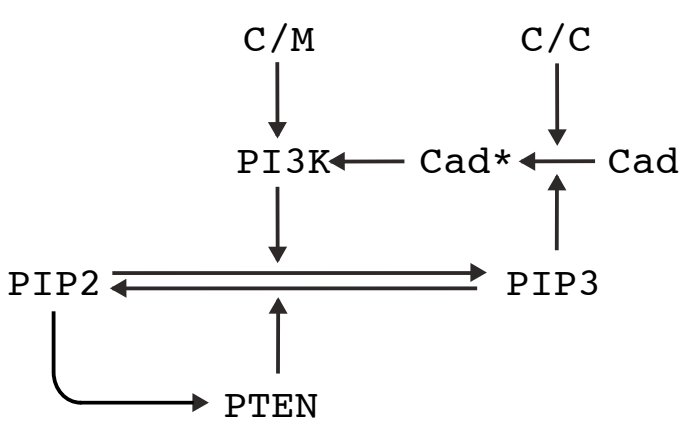

FIG. 2: Reactions scheme of the epithelial polarization network. The core players are the PI3K and PTEN enzymes, which respectively catalyze the switch of the PIP2 lipid into PIP3 and vice versa. PTEN becomes effective upon association to PIP2, while PI3K becomes effective upon association with activated cell-cell adhesive receptors (cadherins, Cad*) or extracellular matrix adhesive receptors $(\mathrm{C} / \mathrm{M})$. To bind PI3K, cadherins (Cad) must be activated $\left(\mathrm{Cad}^{*}\right)$ by linking other cadherins on neighboring cells $(\mathrm{C} / \mathrm{C})$ and by the action of PIP3. The symmetry of PTEN/PI3K in generating PIP2/3 can be broken by cadherins inactivation. The PIP2PTEN feedback loop may then locally prevail, originating the chemical polarization of the cell.

ically found in high curvature membrane regions, especially in correspondence of multiple cell-cell contacts (cellular vertex) 2 away from the extracellular matrix (Fig. 1). We observe here that cell-cell contacts can be broken [14] as soon as the adhesion energy $W_{\mathrm{a}}$ (i.e. the energy per unit area needed to break cadherin contacts) becomes comparable to the elastic energy stored in the cell membrane in high curvature regions, such as at the confluence of several cells (Fig. 1 $\mathrm{a}, \mathrm{b}$ ). Since the elastic energy per unit area $W_{\mathrm{e}}$ stored in the membrane is [15]17.

$$
W_{\mathrm{e}}=\kappa / 2 r^{2},
$$

where $\kappa$ is the membrane bending rigidity and $r$ the local curvature radius [16, the condition $W_{\mathrm{a}} \sim W_{\mathrm{e}}$ allows to estimate the critical curvature radius $r_{\mathrm{a}}$ where cell-cell contacts start being disrupted as [17]

$$
r_{\mathrm{a}} \simeq \sqrt{\kappa / 2 W_{\mathrm{a}}} .
$$

The critical value $r_{\mathrm{a}}$ can be easily estimated. In eukaryotic cells, the typical adhesion energy of cadherin contacts is $W_{\mathrm{a}} \simeq 10^{-11} \mu \mathrm{J} / \mu \mathrm{m}^{2}$ [17, while the typical bending rigidity is $\kappa \simeq 400 k_{\mathrm{B}} T \simeq 16 \times 10^{-13} \mu \mathrm{J}$ [17], giving [37]

$$
r_{\mathrm{a}} \simeq 0.3 \mu \mathrm{m} .
$$

According to the above estimate, when the number of cells in the initial aggregate increases up to the 5-6 cell stage, at the cell convergence points (see Fig. 1b) the membrane curvature increases as well and, especially in 
areas not in contact with the extracellular matrix, cadherin bridges are subject to forces that can disrupt links. By such a mechanism, a local opening of cell-cell contacts breaks the PIP3-PI3K feedback loop and induces a local unbalance towards PIP2 formation (Fig. 2 and a germ of the PIP2-rich phase can be ushered in (Fig. 10).

In Sect. III we show that only germs of the PIP2-rich phase larger than a treshold radius $r_{\text {thr }}$ actually survive and grow. This fact suggests that although the uniform PIP3-rich phase is not the more stable state for the signaling network, a polarized state characterized by the coexistence of the PIP3-rich and the PIP2-rich phase may be reached only by overcoming a barrier in a suitably defined effective energy (see Sect. IV for a detailed discussion of this point). Therefore, we are faced with the following physical picture: if elastic forces due to high membrane curvature in the region of cell convergence (cellular vertex) trigger disruption of cadherin links in a region of size larger than $r_{\mathrm{thr}}$, the PIP2-rich patch grows favoring further breaking of cadherin links (Fig. 2) and the formation of a lumen. Thus, for the process of lumen formation to start, it is necessary that the local curvature radius in the cellular vertex satisfies

$$
r_{\mathrm{a}} \gtrsim r_{\mathrm{thr}}
$$

The growth of the PIP2-patch and lumen slows down and eventually comes to a stop as soon as cytosolic PTEN is depleted. This way, at the end of the process the cell reaches a stable polarized state characterized by the coexistence of the PIP2-rich and the PIP3-rich phase [18, 19], and a lumen coinciding with the PIP2rich phase is formed.

\section{SIMULATIONS.}

In this Section we investigate on quantitative grounds the above described scenario of polarization.

Since the chemical reaction and diffusion processes are intrisically noisy, we simulate the corresponding dynamics by a stochastic algorithm, using realistic values for reaction and diffusion rates. We can check this way that noise alone is not sufficient here to overcome the energy barrier separating the uniform and polarized state in observational times, if an initial PIP2 seed of size larger than $r_{\text {thr }}$ is not created by an external interaction.

We represent the plasmamembrane by a lattice of $\mathcal{N}=10242$ (mostly hexagonal) sites of area $\sigma \sim S / \mathcal{N} \sim$ $(0.1 \mu \mathrm{m})^{2}$ on a sphere surface $S=4 \pi R^{2}$ with radius $R=5 \mu \mathrm{m}$ (Fig. 3 ). Each site is populated by a number of molecules of the chemical factors and their dynamics is described by standard master equations. For instance, the PIP2 $\rightarrow$ PIP3 process is described by

$$
\begin{aligned}
& \partial_{t} P\left(N_{\mathrm{PIP}_{2}}, N_{\mathrm{PIP}_{3}}, \ldots\right)= \\
+ & W\left(\mathrm{PIP}_{3} \rightarrow \mathrm{PIP}_{2}\right) P\left(N_{\mathrm{PIP}_{2}}-1, N_{\mathrm{PIP}_{3}}+1, \ldots\right) \\
- & W\left(\mathrm{PIP}_{2} \rightarrow \mathrm{PIP}_{3}\right) P\left(N_{\mathrm{PIP}_{2}}, N_{\mathrm{PIP}_{3}}, \ldots\right),
\end{aligned}
$$

\begin{tabular}{|c|c|c|}
\hline Reaction & $W$ & Rate constants \\
\hline $\mathrm{PIP} 2 \rightarrow \mathrm{PIP} 3$ & $k_{1} N_{\mathrm{PIP} 2} N_{\mathrm{PTEN}} /\left(K+N_{\mathrm{PIP} 2}\right)$ & $k_{1}=1, K=50$ \\
\hline $\mathrm{PIP} 3 \rightarrow \mathrm{PIP} 2$ & $k_{2} N_{\mathrm{PIP} 3} N_{\mathrm{PI} 3 \mathrm{~K}} /\left(K+N_{\mathrm{PIP} 3}\right)$ & $k_{2}=0.5, K=50$ \\
\hline PTEN $\rightarrow$ PTEN $^{*}$ & $k_{3} N_{\mathrm{PTEN}} N_{\mathrm{PIP} 2}$ & $k_{3}=2 \cdot 10^{-5}$ \\
\hline $\mathrm{PTEN}^{*} \rightarrow$ PTEN & $k_{4} N_{\text {PTEN }} *$ & $k_{4}=0.5$ \\
\hline $\mathrm{PI} 3 \mathrm{~K} \rightarrow{\mathrm{PI} 3 \mathrm{~K}^{*}}^{*}$ & $k_{5} N_{\mathrm{PI} 3 \mathrm{~K}} N_{\mathrm{Cad} *}$ & $k_{5}=2 \cdot 10^{-5}$ \\
\hline 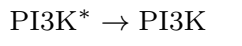 & $k_{6} N_{\mathrm{PI} 3 \mathrm{~K}^{*}}$ & $k_{6}=0.1$ \\
\hline $\mathrm{Cad} \rightarrow \mathrm{Cad}^{*}$ & $k_{7} N_{\text {Cad }} N_{\text {PIP3 }}$ & $k_{7}=2 \cdot 10^{-5}$ \\
\hline $\mathrm{Cad}^{*} \rightarrow \mathrm{Cad}$ & $k_{8} N_{\mathrm{Cad}^{*}}$ & $k_{8}=0.5$ \\
\hline
\end{tabular}

TABLE I: List of chemical reactions involved in epithelial polarization and their corresponding rates. $\mathrm{X}^{*}$ denotes the membrane-bound, activated form of molecule X. Rate constants $k$ are given in $\mathrm{s}^{-1}$. Activation rates can be transformed to $(\mathrm{s} \mathrm{M})^{-1}$ units multiplying by Avogadro's number $N_{\mathrm{A}}$. Michaelis-Menten constants $K$ are pure numbers and can be transformed to $\mathrm{M}$ units dividing by $N_{\mathrm{A}} / \mathcal{N}$. For the rate constants values see references in 20].

where $P\left(N_{\mathrm{X}}, \ldots\right)$ is the probability to have at time $t$ a number $N_{\mathrm{X}}$ of type $\mathrm{X}$ molecules at a given site (say, $i$.) The list of relevant reactions with their corresponding rates $W$ is given in Table I 38. PIP2/3 diffusion is described by random jumps of a molecule from site $i$ to its neighboring site $j$ with rate $W(i \rightarrow j)=N_{\mathrm{X}} D / \sigma$, where $D=0.5 \mu \mathrm{m}^{2} / \mathrm{s}$ is phospholipid diffusivity [20]. Since the diffusivity of cytosolic enzymes (PI3K/PTEN) is much larger than that of membrane pospholipids [20], their distribution in the cytosol is treated as uniform.

For the simulations we use a variation of Gillespie algorithm 21] taking into account the spatial non-uniformity of the system. At time zero, a random number is generated to determine the next reaction or elementary diffusion process to occur, with a probability proportional to the corresponding $W$ factor from Table $[$. Then, time is advanced as a Poisson process with rate again determined by the $W$ factors. These steps are repeated iteratively until the desired simulation time is reached.
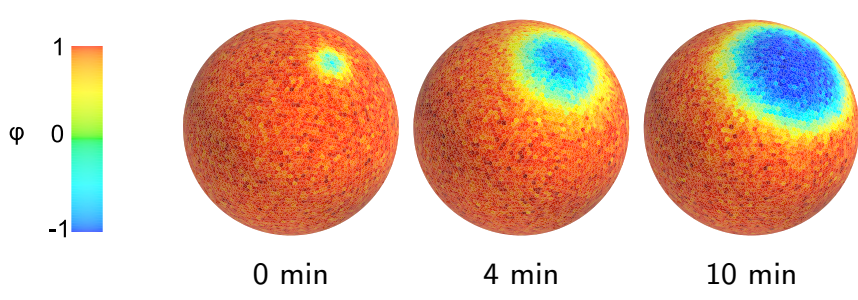

FIG. 3: (Color online) Growth of the PIP2-rich phase (blue upper patch). The color scale shows the gradation of PIP2 content: $\varphi$ is the relative concentration difference between PIP3 and PIP2 at a given site. The system at initial time is in a uniform PIP3-rich phase, apart from an initial PIP2rich seed germ of size $r_{0}$ larger than the threshold radius $r_{\text {thr }}$ (small circle.) After 4 minutes a PIP2-rich patch becomes apparent and its radius saturates after approximately 10 minutes to the equilibrium value $r_{\text {eq }}$. 


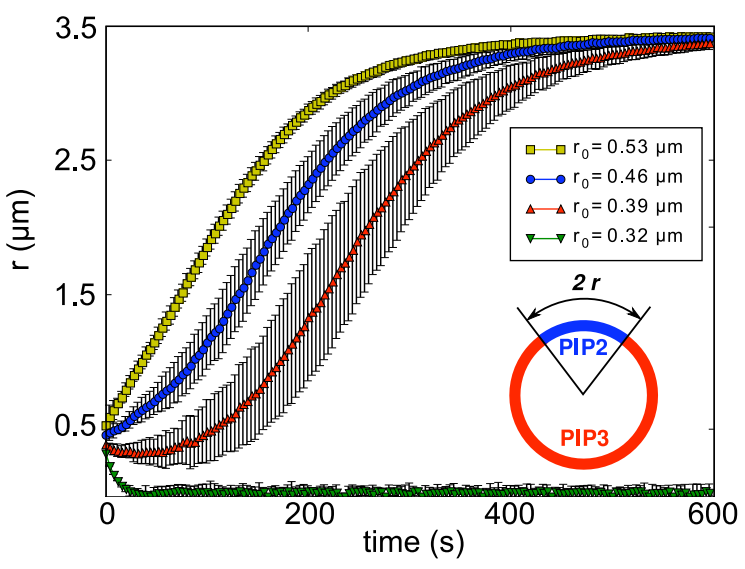

FIG. 4: (Color online) Growth of the PIP2-rich phase induced by a germ of initial radius $r_{0}$. Germs with $r_{0}$ smaller than a threshold radius $r_{\text {thr }} \sim 0.3 \mu \mathrm{m}$ are melted by diffusion, while larger germs grow to the equilibrium value $r_{\text {eq }}$. Error bars show standard deviations computed over $n=50$ different random realizations of the process.

We suppose that a circular PIP2-rich patch of radius $r_{0}$ is initially formed in the sea of the PIP3-rich phase [39] and investigate its dynamics to check whether a stable polarization state is attained (Fig. 3). Fig. 4 shows the time evolution of circular patches of different initial radii $r_{0}$. Patches smaller than a threshold radius $r_{\mathrm{thr}} \sim 0.3 \mu \mathrm{m}$ are dissolved by diffusion and thermal processes, and do not impair the stability of the uniform PIP3-rich phase. Conversely, patches larger than $r_{\text {thr }}$ grow in time triggering the separation of the cell surface in a PIP2-rich and a PIP3-rich region and eventually reach an equilibrium (Fig. 3). Notably, the threshold radius $r_{\text {thr }} \sim 0.3 \mu \mathrm{m}$ derived from the above calculation is consistent with the previously independently derived value for the adhesion radius, $r_{\mathrm{a}}$. The two phases are divided by an interface of characteristic width $\delta \sim \sqrt{D / k_{\mathrm{c}}} \sim 1 \mu \mathrm{m}$ [19, where $k_{\mathrm{c}}$ is of the order of the catalytic constants of the two catalytic reactions of Table I (first two rows).

The kinetic of this heterogeneous nucleation process can be understood in terms of non-equilibrium, reactiondiffusion stochastic dynamics. In reaction-diffusion systems instabilities are often produced by Turing's mechanism 22]. Here we find that pattern formation starting from a locally stable homogeneous state is triggered by a local perturbation by a nucleation center of size $r_{0}$ larger than a critical size $r_{c}[19,23,25$.

Fig. 5 shows that the equilibrium size $r_{e q}$ of the PIP2rich patch, and therefore of the lumen, is controlled by the number of PI3K and PTEN molecules. In the absence of any limiting mechanism, the growth of the PIP2-rich patch would in fact lead to a PIP2-rich phase completely invading the cell surface. However, due to the coupling to a finite PTEN and PI3K reservoir, the system selftunes to a phase-coexistence state and the process stops when the PIP2-rich patch reaches the equilibrium size

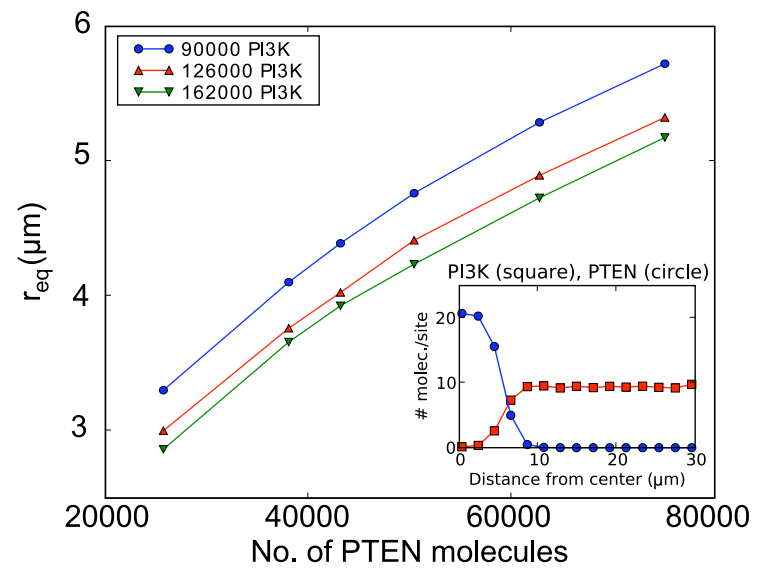

FIG. 5: (Color online) The equilibrium lumen size $r_{\mathrm{eq}}$ is an increasing function of the relative number of PI3K and PTEN molecules. Inset: radial distribution of PI3K (red squares, $N_{\mathrm{PI} 3 \mathrm{~K}}=1.26 \cdot 10^{5}$ molecules) and PTEN (blue circles, $N_{\text {PTEN }}=0.43 \cdot 10^{5}$ molecules $)$, from the patch center, at equilibrium.

$r_{e q}$ [18 20]. Interestingly, the fact that the size of the PIP2-rich patch, and consequently of the lumen, is controlled by the precise number of PTEN molecules, is in qualitative agreement with the observation that deletion of a single PTEN allele can interfere with the polarization process [26].

The observation in the present scenario of a comparatively large threshold radius, of the order of one tenth of the cell size, suggests the existence of a correspondingly large barrier of effective energy dividing the uniform state from the phase-separated one. This prevents thermal and chemical noise from triggering spontaneous symmetry breaking and lumen formation. However, an external mechanical action creating a sizeable PIP2-rich patch, due to the presence of localized regions of high membrane-curvature, can overcome the barrier and start polarization [40]. Our picture also explains tight junction localization. Experimental data show that the stable binding of tight junction proteins to the membrane requires both a protein complex named PAR3-PAR6, which is localized in the PIP2-rich phase by a chain of reactions, and cell-cell contacts, which are maintained only in the PIP3-rich phase [27]. The spontaneous aggregation of tight junctions is thus constrained by a biochemical logical AND to take place only on the ring-shaped boundary separating the PIP2-rich from the PIP3-rich phase.

\section{MEAN-FIELD}

In this Section we show that the results of the simulations can be conveniently interpreted in terms of an effective mean-field model, following the approach detailed in Refs. [19, 25.

Fast diffusion of PI3K and PTEN enzymes in the cy- 
tosol, and the conservation law $[\mathrm{PIP} 2]+[\mathrm{PIP} 3]=c$ allow to effectively describe the state of the cell membrane in terms of the configuration of the single-component concentration field [19, 25]:

$$
\varphi=[\mathrm{PIP} 3]-[\mathrm{PIP} 2] .
$$

The resulting effective equation for $\varphi$ can be set in the simple Landau-Ginzburg form:

$$
\partial_{t} \varphi=D \nabla^{2} \varphi+V^{\prime}(\varphi)+\xi,
$$

complemented by an integral costraint expressing the coupling of the concentration field $\varphi$ to the reservoir of free cytsolic enzymes (see Ref.s [19, 25] and Supplementary Information in 20].) In Eq. (7), $D$ is the diffusivity of lipids on the cell membrane, $V(\varphi)$ is an effective potential, and $\xi$ is a stochastic term taking into account the effect of thermal and chemical noise.

The mean-field effective potential $V(\varphi)$ can be easily derived, via a quadratic approximation, from the stochastic model described in Sect. III under the assumption that the cytosolic PI3K, PTEN and Cad fields are in approximate equilibrium with the membrane PIP2 and PIP3 fields, and therefore "slaved" to the $\varphi$ field [19, 25]:

$$
V^{\prime}(\varphi)=-\alpha \frac{c^{2}-\varphi^{2}}{2 K+c+\varphi}+\alpha^{\prime} \frac{c^{2}-\varphi^{2}}{2 K+c-\varphi}
$$

where

$$
\begin{aligned}
\alpha & =\frac{k_{2} k_{3}}{k_{4}}[\mathrm{PTEN}]_{\text {free }}, \\
\alpha^{\prime} & =\frac{k_{1} k_{5}}{k_{6}}[\mathrm{PI} 3 \mathrm{~K}]_{\text {free }} \cdot \frac{k_{7}}{k_{8}}[\mathrm{Cad}]_{\text {free }} .
\end{aligned}
$$

The terms in the r.h.s. of Eq. (8) describe respectively conversion of PIP3 into PIP2 due to the action of PTEN, and conversion of PIP2 into PIP3 due to the action of PI3K activated by cadherins (Fig. 2). The quadratic terms $\propto c^{2}-\varphi^{2}$ encode respectively the PIP2 $\rightarrow$ PTEN and the PIP $3 \rightarrow$ Cad $\rightarrow$ PI3K feedback loops (Fig. 2). In particular, $\alpha^{\prime}=0$ when cadherin links are broken.

In a wide region of parameter space around the realistic parameter values from Table [. the effective potential $V(\varphi)$ is bistable (Fig. 6; for a detailed description of the bistability region, see Ref. [28). The two potential wells in Fig. 6 correspond to a stable PIP2-rich and a metastable PIP3-rich phase, separated by an energy barrier $\Delta V$.

The mean-field model $(7,8)$ and the bistability of the effective potential $V(\varphi)$ provide an interpretation to the simulation results, showing that the stable polarized state characterized by the coexistence of PIP2 and PIP3 in complementary regions is separated from the metastable PIP3-rich phase by an effective energy barrier $\Delta V$. According to the theory of Landau-Ginzburg equation, PIP2-rich seeds larger than a critical value are bound to expand in the PIP3-rich sea with a velocity proportional to $1 / \Delta V$ [23.

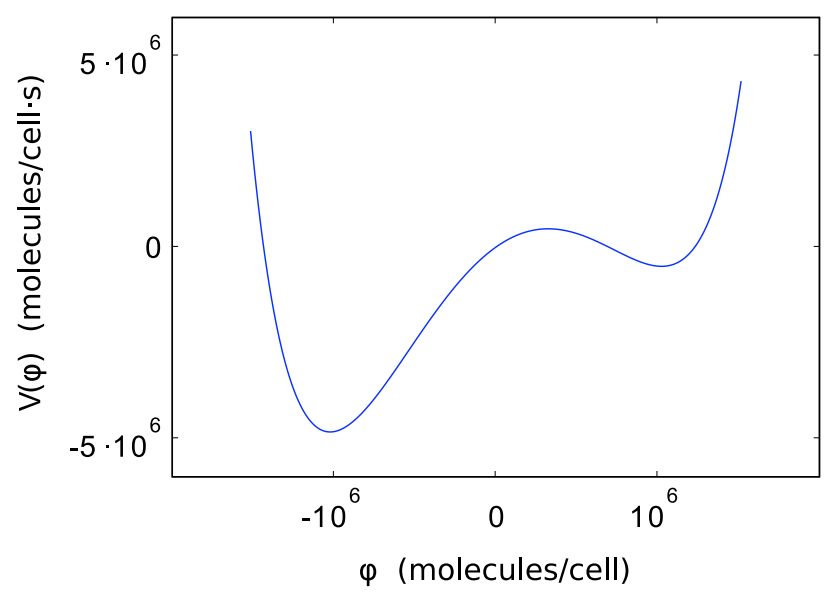

FIG. 6: (Color online) Graph of the effective potential $V(\varphi)$ defined by 8910 , where the cytosolic values $[\mathrm{PTEN}]_{\text {free }} \simeq$ $1.1 \cdot 10^{4},[\overline{\mathrm{PI} 3 \mathrm{~K}}]_{\text {free }} \simeq 3.7 \cdot 10^{4},[\mathrm{Cad}]_{\text {free }} \simeq 5.4 \cdot 10^{3}$ (molecules/cell) were computed from simulations of the stochastic model described in Sect. III at time $t=30 \mathrm{~s}$, after the fast equilibration of the corresponding cytosolic pools of enzymatic factors with the initial configuration of the $\varphi$ field. The effective potential $V(\varphi)$ has two minima, the left hand one corresponding to a stable PIP2-rich and the right hand one corresponding to a metastable PIP3-rich phase. The two phases are separated by an effective energy barrier.

The cytosolic concentrations $[\mathrm{PI} 3 \mathrm{~K}]_{\text {free }},[\mathrm{PTEN}]_{\text {free }}$, and $[\mathrm{Cad}]_{\text {free }}$ appearing in (8) may be expressed as integrals of the concentration field $\varphi$ [25, 28. The resulting global coupling has the effect of driving dynamically the cell membrane towards an equilibrium polarized state where the PIP2-rich and PIP3-rich phases coexist: the growth of the PIP2-rich phase "eats up" free PTEN molecules from the cytosol, decreasing $\Delta V$ until phase coexistence is reached [25, 28. This process may be understood via a simple physical analogy with the non-equilibrium process taking place during the liquidvapor transition in a sealed vessel: there, the rise of the vapor pressure (which in our analogy corresponds to the number of cytosolic enzymes) provides a negative feedback, slowing down the growth of the vapor phase and eventually leading the system to a state of phase coexistence. The main difference between the two systems is that in the liquid-vapor transition a local conservation law holds for the particle field, while in the growth of signaling domains on the cell membrane the $\varphi$ field satisfies only an approximate global constraint 25] encoded in the integral expressions for the coefficients $\alpha, \alpha^{\prime}$ appearing in 89910.

It is worth observing here that while spontaneous polarization in eukaryotic directional sensing [20] can be described in terms of an homogeneous nucleation process, whereby seeds of a PIP3-rich phase are created by thermal and chemical noise in the sea of the PIP2-rich phase and grow by a coarsening process [19, 25, the present 
picture of epithelial polarization reminds instead heterogeneous nucleation, i.e. a situation where the effective potential barrier $\Delta V$ is so high that spontaneous nucleation does not occur in typical observational times, and needs to be triggered by the introduction of a large enough nucleation germ.

\section{DISCUSSION}

We have shown that a simple symmetry breaking mechanism, informed with the recently discovered biochemical and biophysical details of the system, accounts for a wealth of morphogenetic processes in epithelial polarization. The model makes specific predictions on the dependence of the threshold radius, lumen size, tight junction positioning and width, on the biochemical system parameters. Our results shed light on the role of PTEN as a tumor suppressor protein, whose expression levels are known to be critical to prevent the onset of cancer. In particular, our models predicts that by decreasing the number of PTEN molecules, the lumen size should decrease, and for very low PTEN levels no lumen at all should form. The experimental validation of the model could be performed by genetic manipulation of the amounts or activity of cadherins, PI3K and PTEN.

We have also shown that curvature induced forces are a very plausible candidate for triggering the symmetry breaking process at the right time. This could be verified experimentally by trying to induce localized formation of a growing PIP2 patch and lumen by mechanically breaking adhesion bonds in localized regions of the membrane of epithelial cells surrounded by extracellular matrix. Under these conditions, our model predicts that only breaking adhesion bonds in regions larger than $r_{\text {thr }}$ should induce the formation of a growing patch of the PIP2-rich phase, while smaller PIP2-rich patches, induced by breaking adhesive bonds on smaller regions, should shrink spontaneously.

Interestingly, the bistable PI3K-PTEN module here described plays also a key role in chemotaxis, where PI3K is initially activated by chemotactic receptors (see the review [29] and ref.s therein) rather than by adhesive receptors. While experimental evidences and our results suggest that epithelial polarization is induced by a nucleation center of the PIP2-rich phase generated by mechanical forces, the polarization of migrating cells is likely to be triggered by spontaneous fluctuations in PIP3/PI3K levels [19].

PIP3 localization also regulates chemotactic polarization [19, 20] and cell spindle orientation [30. The similarity underlying the mechanisms in these very different aspects of cell life hints to the possibility that phase separation phenomena might have a general role in the cell [19, 20] and in its nucleus [31, 32. The principles emerging here could explain in a universal way the deep analogies observed in a variety of cellular processes involving spatial polarity formation [1].

\section{ACKNOWLEDGMENTS}

We thank G. Boffetta for discussions, hospitality at ISAC-CNR and access to computational facilities, and S. Vegetti for suggestions. This work was partially supported by Telethon-Italy GGP04127, AIRC, MIUR (PRIN 2007BMZ8WA), Regione Piemonte, PRESTO, Fondazione CRT, Ministero della Salute.
[1] F. Comer and C. Parent, Cell 128, 239 (2007).

[2] F. Martin-Belmonte, A. Gassama, A. Datta, W. Yu, U. Rescher, V. Gerke, and K. Mostov, Cell 128, 383 (2007).

[3] A. Z. Wang, G. K. Ojakian, and W. J. Nelson, J Cell Sci 95 ( Pt 1), 137 (1990).

[4] D. M. Bryant and K. E. Mostov, Nat Rev Mol Cell Biol 9, 887 (2008).

[5] I. Mellman and W. J. Nelson, Nat Rev Mol Cell Biol 9, 833 (2008).

[6] N. van Kampen, Stochastic Processes in Physics and Chemistry (North-Holland, 2007), 3rd ed.

[7] S. Ramanathan, P. B. Detwiler, A. M. Sengupta, and B. I. Shraiman, Biophys J 88, 3063 (2005).

[8] M. Thattai and A. van Oudenaarden, Biophys J 82, 2943 (2002).

[9] J. Paulsson, O. G. Berg, and M. Ehrenberg, Proc Natl Acad Sci U S A 97, 7148 (2000).

[10] V. Kölsch, P. G. Charest, and R. A. Firtel, J Cell Sci 121, 551 (2008).

[11] S. J. Watton and J. Downward, Curr Biol 9, 433 (1999).
[12] A. S. Yap and E. M. Kovacs, J Cell Biol 160, 11 (2003).

[13] L. Landau and E. Lifshitz, Course of Theoretical Physics, vol. 5 (Butterworth-Heinemann, 1980).

[14] R. Kroschewski, News Physiol Sci 19, 61 (2004).

[15] L. Landau and E. Lifshitz, Course of Theoretical Physics, vol. 7 (Butterworth-Heinemann, 1986).

[16] J. Fournier, Soft Matter 3, 883 (2007).

[17] R. Simson, E. Wallraff, J. Faix, J. Niewöhner, G. Gerisch, and E. Sackmann, Biophys J 74, 514 (1998).

[18] T. Ferraro, A. de Candia, A. Gamba, and A. Coniglio, Europh. Lett. 83, 50009 (2008).

[19] A. Gamba, I. Kolokolov, V. Lebedev, and G. Ortenzi, Phys Rev Lett 99, 158101 (2007).

[20] A. Gamba, A. de Candia, S. D. Talia, A. Coniglio, F. Bussolino, and G. Serini, Proc Natl Acad Sci U S A 102, 16927 (2005).

[21] D. T. Gillespie, The Journal of Physical Chemistry 81, 2340 (1977).

[22] A. M. Turing, Phil Trans R Soc Lond B 237, 37 (1952).

[23] A. Bray, Adv. Phys. 43, 357 (1994).

[24] E. Schöll, Stochastic Processes in Physics, Chemistry, 
and Biology (Springer, 2000), pp. 437-451.

[25] A. Gamba, I. Kolokolov, V. Lebedev, and G. Ortenzi, Journal of Statistical Mechanics: Theory and Experiment 2009, P02019 (2009).

[26] A. Gassama-Diagne, W. Yu, M. ter Beest, F. MartinBelmonte, A. Kierbel, J. Engel, and K. Mostov, Nat Cell Biol 8, 963 (2006).

[27] B. Margolis and J.-P. Borg, J Cell Sci 118, 5157 (2005).

[28] A. Gamba, G. Naldi, M. Semplice, G. Serini, and A. Veglio, in preparation (2009).

[29] P. A. Iglesias and P. N. Devreotes, Curr Opin Cell Biol 20, 35 (2008).

[30] F. Toyoshima, S. Matsumura, H. Morimoto, M. Mitsushima, and E. Nishida, Dev Cell 13, 796 (2007).

[31] M. Nicodemi and A. Prisco, Phys. Rev. Lett. 98, 108104 (2007).

[32] M. Nicodemi, B. Panning, and A. Prisco, Genetics 179, 717 (2008).

[33] B. Vanhaesebroeck, S. J. Leevers, K. Ahmadi, J. Timms, R. Katso, P. C. Driscoll, R. Woscholski, P. J. Parker, and M. D. Waterfield, Annu Rev Biochem 70, 535 (2001).

[34] C. L. Carpenter, B. C. Duckworth, K. R. Auger, B. Cohen, B. S. Schaffhausen, and L. C. Cantley, J Biol Chem 265, 19704 (1990).

[35] D. Duguay, R. A. Foty, and M. S. Steinberg, Dev Biol
253, 309 (2003).

[36] B. I. Shraiman, Proc Natl Acad Sci U S A 102, 3318 (2005).

[37] It is worth observing here that since the values $\kappa$ and $W_{\text {a }}$ used for the estimate have been measured on live cells [17, they take effectively into account the mechanical contributions from both the lipid bilayer and the cortical cytoskeleton.

[38] The values for processes involving cadherins are educated guesses since no precise data are available. One order of magnitude changes in these values do not result however in appreciable modifications of the system dynamics.

[39] Where not otherwise stated, the following experimentally realistic values for the initial concentration are used: $[\mathrm{PIP} 2]+[\mathrm{PIP} 3]=10^{6}$ (according to $[33),[\mathrm{PI} 3 \mathrm{~K}]=$ $[\mathrm{Cad}]=10^{5}$ (according to 34 and 35, respectively), $[\mathrm{PTEN}]=0.2 \times 10^{5}$ (molecules/cell). For the PTEN concentration, we assumed the same order of magnitude as in [20] and studied in Fig. 5 the system behaviour on varying the PTEN molecules number. The initial PIP3rich phase is $98 \%$ PIP3, $2 \%$ PIP2.

[40] Interestingly, the role of mechanical forces has been suggested also in other settings of tissue morphogenesis 36. 